\title{
On Preparing Offers for Targeted Development Programs in Housing and Communal Services
}

\author{
Rustam Khayrullin ${ }^{1, *}$ \\ Moscow state university of civil engineering, Yaroslavskoye shosse, 26, Moscow, Russia, 129337
}

\begin{abstract}
The article offers a mathematic development model for economically sound and close to optimum park of technical facilities used in housing and communal services, that implies purchasing new samples of these facilities and upgrade items already existing that have quite low level of technical perfection. A level of technical perfection of the item is a complex (integral) characteristic that relies on the system of indexes that describe its main properties, based on its purpose, and is reflective of its technical progressiveness. Level of technical perfection of the park is a mean weighted sum of technical perfection parameters for all the items in the park. The model is based on algorithm for presentation of technical facilities development park and is made as a standard task of linear programming, solution methods with limitations in the form of equalities and inequalities, including methods of integer linear programming. The criterion used is an indicator that characterize reduction of degradation, a value inverse to the technical perfection level. The author analysed economic expediency of combined purchases and upgrades for various types of technical facilities under various amounts of financing. The article presents results of mathematic model approbation.
\end{abstract}

\section{Introduction}

Housing and communal services (HCS) present one of the most promising sector of Russian economy in terms of increasing power efficiency and energy saving. The data of Russian Federation Ministry of Energy, HSS consumes 16\% of fuel total amount of fuel used in the country.

One of the most promising trends for solving the energy efficiency problem is the one that supported from the 'smart house-smart city-smart country' concept, among other things, in the frames of long-term target programs [1], like «Development of housing and communal services for years 2014-2018», «Development strategy for housing and communal services of Russian Federation by year 2020» and other territorial programs of HCS development.

Implementation of these programs requires introducing and operation of instruments, tools and equipment (ITE) of sufficiently high technical perfection, targeted management of instruments, tools and equipment park (ITEP) used in HCS sector.

Topical is a task of developing a method that can obtain rational structure of ITEP, develop versions of ITEP re-equipment in accordance with modern requirements, manage

\footnotetext{
* Corresponding author: KhairullinRZ@mgsu.ru
} 
purchasing and upgrade of facilities samples both for making offers for targeted development programs and for control of implementation of these programs.

At the same time, the method should be quite easy to use, be applicable for tasks of various dimensionality, fit for optimum management of the whole ITEP and/or for its part.

The article presents the development results of methodical approaches [2-4] to ITE used by HCS sector. The article offers the mathematic model for optimum strategies of purchasing and upgrade that assure maximum level of technical perfection (minimum level of degradation) for the planned date, at the required level of ITE, with regard to limitations for production capabilities and total cost of purchases and upgrades.

[2] implements the approach to finding a technical perfection of the ITE sample based on comparison of main technical and operation parameters the group of experts made between the sample researched and some base sample. To assess technical perfection of the ITE sample, the article uses compression of indicators like reliability, accuracy, automation level, autonomy etc. Technical perfection level of ITE park is generally understood as a mean weighted sum of technical perfection indicators of all items that make ITE park.

In [4], classic theory of optimal control and approximate methods for solution of optimization tasks [5-7] contribute to posing and solving the task of targeted management of efficiency indicators for metrological equipment park.

Direct use of [4] methods and results that would model close to optimum HCS-used HITEP renewal processes is hindered by absence of models for joint records of purchase and upgrade, with regard to their time and limited production capacities of instrumentmaking industry, as well as of specific character of linear programming problem (LPP), particularly, in the fact that LPP matrix is inherently heterogeneous and highly dimensional [8].

The article assumes the modernization takes several years and receives certain amounts of financing throughout its time. Upgraded technical facility takes a year to be put in operation. Unlike upgrade, purchase of new ITE sample is instant. Purchased sample is put in operation in the year of when it was bought.

\section{Posing the task for joint management of purchases and upgrades}

Let's denote:

$T Z$ - number of ITE types that can be purchased, $t z=1,2, \ldots, T Z$,

$T M$ - number of ITE types that can be upgraded, $t m=1,2, \ldots, T M$,

$k z^{t z}$ - number of park ITE samples of type ( $t z, t z=1,2, \ldots, T Z$ ), having degradation levels of $n z^{t z}$, instead of which new ITE samples may be purchased,

$\mathrm{km}^{\mathrm{tm}}$ - number of park ITE samples of type $(\mathrm{tm}, \mathrm{tm}=1,2, \ldots, \mathrm{TM})$ having degradation levels of $\mathrm{nm}^{\mathrm{tm}}$, that can be upgraded,

$p$ - duration of planning period,

$p m$ - duration of upgrade,

$C Z_{i}^{t z}$ - costs of $t z$ - type ITE samples purchased in $i$ year,

$C M_{i}^{t m}$ - costs of $t m$-type ITE samples upgraded in $i$ year, 
$S_{t o t}=C_{1}+C_{2}+\ldots+C_{p}$ - limitation of total cost for all purchased and upgraded ITE samples for the whole period of planning.

$\left(P_{i}^{t m}, t m=1,2, \ldots, T M, i=1,2, \ldots, p\right)$ - limitation for production capacities of instrument-making industry;

$C_{\mathrm{deg}}^{t z}$ - upper limitation for $t z$ - type ITE level of degradation,

$C_{\mathrm{deg}}^{t m}$ - upper limitation for $t m$ - type ITE level of degradation,

$\alpha^{t z}, \beta^{t m}$ - weight factors with regard to importance for upgrade of various ITE types.

Now, let's introduce variables:

$x_{i}^{t k}$ - number of $t k$-type ITE samples purchased in $i$ year,

$z_{i}^{t m}$ - number of $t m$-type ITE samples upgraded in $i$ year.

Formulate the task of ITEP renewal as follows: we are to obtain $x_{i}^{t z}, z_{i}^{t m}$ so that the general level of ITEP degradation would take on minimum values:

$$
\begin{aligned}
F & =\sum_{t z=1}^{T Z} \alpha^{t z} \cdot k z^{t z} \cdot n z^{t z}+\sum_{t m=1}^{T M} \beta^{t m} \cdot k m^{t m} \cdot n m^{t m}- \\
& -\sum_{t z=1}^{T Z} \sum_{i=1}^{p} \alpha^{t z} \cdot x_{i}^{t z} \cdot n z^{t z}-\sum_{t m=1}^{T M} \sum_{i=1}^{p} \beta^{t m} \cdot z_{i}^{t m} \cdot n m^{t m} \rightarrow \min ,
\end{aligned}
$$

with limitations for the number of various types ITE to be purchased (quantity of various types ITEs:

$$
\sum_{i=1}^{p} x_{i}^{t z} \leq k z^{t z}, t z=1,2, \ldots, T Z,
$$

and quantity of ITE samples to be upgraded:

$$
\sum_{i=1}^{p} z_{i}^{t m} \leq k m^{t m}, t m=1,2, \ldots, T M
$$

It is presumed that in every year of planning $i=(1, \ldots, p)$ total cost of purchases and upgrades is limited by the amount of funds $C_{1}, C_{2}, \ldots, C_{p}$ allocated for the year planned:

$$
\begin{gathered}
\sum_{t z=1}^{T Z} C Z_{1}^{t z} x_{1}^{t z}+\sum_{t m=1}^{T M} C M_{1}^{t m} z_{p m+1}^{t m} \leq C_{1}, \\
\sum_{t z=1}^{T Z} C Z_{2}^{t z} x_{2}^{t z}+\sum_{t m=1}^{T M} C M_{1}^{t m} z_{p m+1}^{t m}+\sum_{t m=1}^{T M} C M_{2}^{t m} z_{p m+2}^{t m} \leq C_{2}, \\
\ldots \ldots \\
\sum_{t z=1}^{T Z} C Z_{i}^{t z} x_{i}^{t z}+\sum_{j=1}^{i} \sum_{t m=1}^{T M} C M_{j}^{t m} z_{p m+j}^{t m} \leq C_{i}, i=1,2, \ldots, p m ;
\end{gathered}
$$




$$
\begin{gathered}
\sum_{t z=1}^{T Z} C Z_{i}^{t z} x_{i}^{t z}+\sum_{j=1}^{p m} \sum_{t m=1}^{T M} C M_{i+j-p m}^{t m} z_{i+j}^{t m} \leq C_{i}, i=p m+1, \ldots, p-p m ; \\
\ldots \\
\sum_{t z=1}^{T Z} C Z_{i}^{t z} x_{i}^{t z}+\sum_{j=i}^{p-1} \sum_{t m=1}^{T M} C M_{j-p m+1}^{t m} z_{j+1}^{t m} \leq C_{i}, i=p-p m+1, p-p m+2, \ldots, p-1 ; \\
\ldots \ldots \\
\sum_{t z=1}^{T Z} C Z_{p-2}^{t z} x_{p-2}^{t z}+\sum_{t m=1}^{T M} C M_{p-p m-1}^{t m} z_{p-1}^{t m}+\sum_{t m=1}^{T M} C M_{p-p m}^{t m} z_{p}^{t m} \leq C_{p-2}, \\
\sum_{t z=1}^{T Z} C Z_{p-1}^{t z} x_{p-1}^{t z}+\sum_{t m=1}^{T M} S M_{p-p m}^{t m} z_{p}^{t m} \leq C_{p-1}, \\
\sum_{t z=1}^{T Z} C Z_{p}^{t z} x_{p}^{t z} \leq C_{p} ;
\end{gathered}
$$

with limitations to industry production capacities in upgrade of ITE samples:

$$
z_{i}^{t m} \leq P_{i}^{t m}, i=p m+1, p m+2, \ldots, p
$$

and limitations for degradation level of each ITE type:

$$
\begin{gathered}
\alpha^{t z} \cdot k z^{t z} \cdot n z^{t z}-\sum_{i=1}^{p} \alpha^{t z} \cdot x_{i}^{t z} \cdot n z^{t z} \leq C_{\mathrm{deg}}^{t z}, \quad t z=1,2, \ldots, T Z \\
\beta^{t m} \cdot k m^{t m} \cdot n m^{t m}-\sum_{i=1}^{p} \beta^{t m} \cdot z_{i}^{t m} \cdot n m^{t m} \leq C_{\mathrm{deg}}^{t m}, \quad t m=1,2, \ldots, T M
\end{gathered}
$$

Note that (1-7) is a task of integer linear programming.

Moreover, inequalities system (4) describes financial limitations with the assumption that various types of ITE have the same duration. Typical features of forming the LPP matrix for different duration of upgrade for various ITE types are described below.

\section{Approbation results}

Initial data: ITE assortment, averaged costs of purchases and upgrades, planning period, upgrade duration, financing distribution broken down by years of planning, production limitations, limitations of allowable level of degradation for each ITE type, $T Z=2$, $T M=1, \alpha^{1}=1, \alpha^{2}=1, \beta^{1}=1, k_{1}^{1}=5, n_{1}^{1}=1,1, k_{1}^{2}=15, n_{1}^{2}=1,3, k_{1}^{3}=10$, $n_{1}^{3}=1,1, C Z^{1}=10, C Z^{2}=15, C M^{1}=3, p m=4, p=20$.

Calculations were made for different amounts of financing $S_{t o t}$, with $C_{1}=C_{2}=\ldots=C_{p}=S_{t o t} / p$. The article presents results of dimensionless calculations for limited ITEP used for HCS service automation: detectors, controllers and 
automated work places. The predictions proved the limitations (5)-(7) fulfil as a strict inequality, as the model was approbated for the ITEP part with the highest level of degradation. Purchase cost was assumed to be not related to the year of planning.

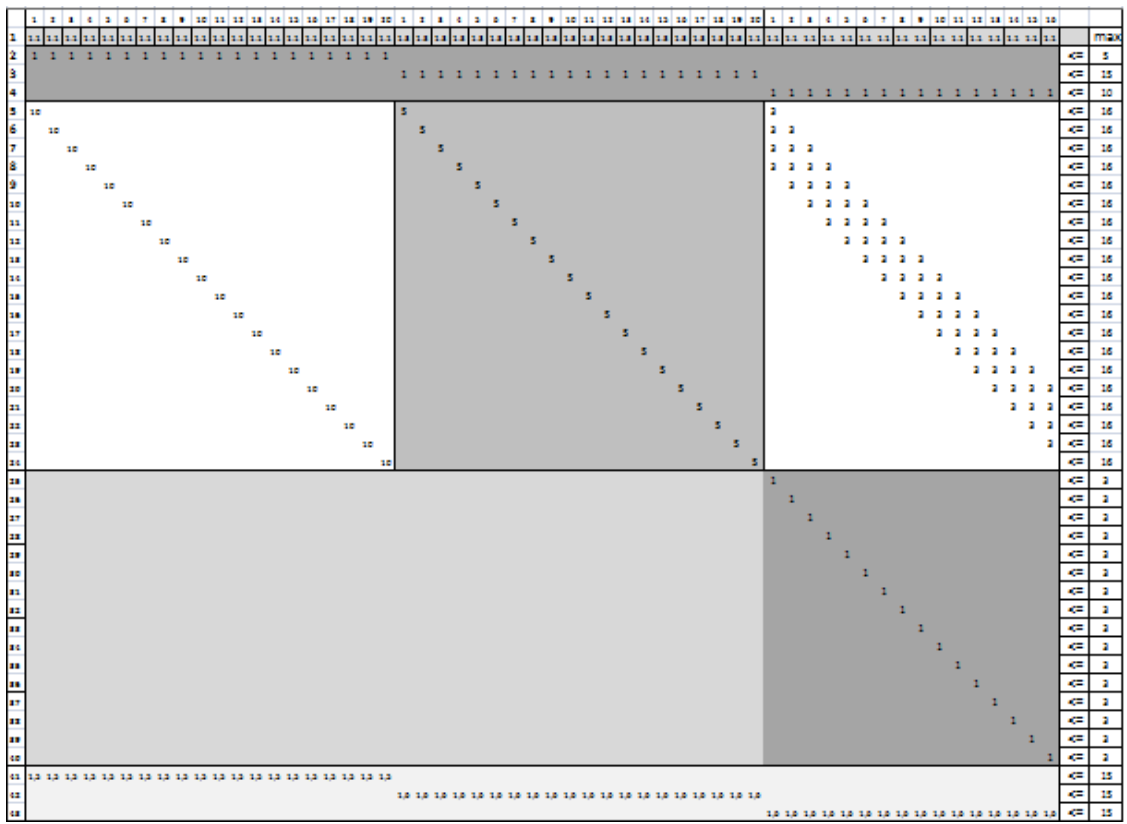

Figure 1. Structure of matrix of line program problem

The view of LPP matrix [4, 7] is shown at Fig. 1. Note that the matrix has a block structure and most of the matrix elements are equal to zero. The first line of the matrix carries objective functions coefficients. Next three lines represent inequalities limited by three types of ITE samples. Below, there are five blocks, with three of them being diagonal. First two blocks represent the purchases of two ITE types. The third block that represents includes two column vectors with nonzero elements arranged in a row. The fourth block consists of zeroes. The fifth, diagonal-shaped block, represents limitations for industry production capacities. Last three rows of the matrix represent limitations for degradation level allowable for each ITE type.

Note that the amount of nonzero elements in the columns of the blocks that represent upgrade is the same as the upgrade period in years. In case various ITE types are upgraded in the same year, and/or various ITE samples having various duration of upgrade, LPP matrix contains several blocks of type three with various amount of nonzero column and various amount of nonzero elements in appropriate columns.

The solution of linear programing employed both Homory method and standard simplex method. The calculations proved that higher amount of financing reduces ITEP degradation level from 31.6 to zero by almost linear law. The explanation is that the degradation indicators for various ITE types are very close to each other.

Amount of financing $S_{\text {tot }}=395$ is sufficient for complete renewal of ITEP. With lower financing, complete renewal of ITEP is impossible.

Fig. 2 offers optimum purchase-upgrade combinations by years of planning for various types of ETI. 
If financing is low, it is wise to purchase of first type ITEPs first: ITE-1, and funds remained should be used to upgrade ITE-U. Medium-size financing first pays the purchase of ITE-1 until complete renewal of its park, then pays upgrade of ITE-U until complete renewal of its park, and, at last, ITE-2 is purchased. When the financing is large enough, purchase of ITE-1 is financed first, and, before renewal of ITE-1 is completed, some funds are allocated for ITE-U renewal. At last, after complete renewal of ITE-1 park and partial renewal of ITE-U, purchase of ITE-2 is financed.
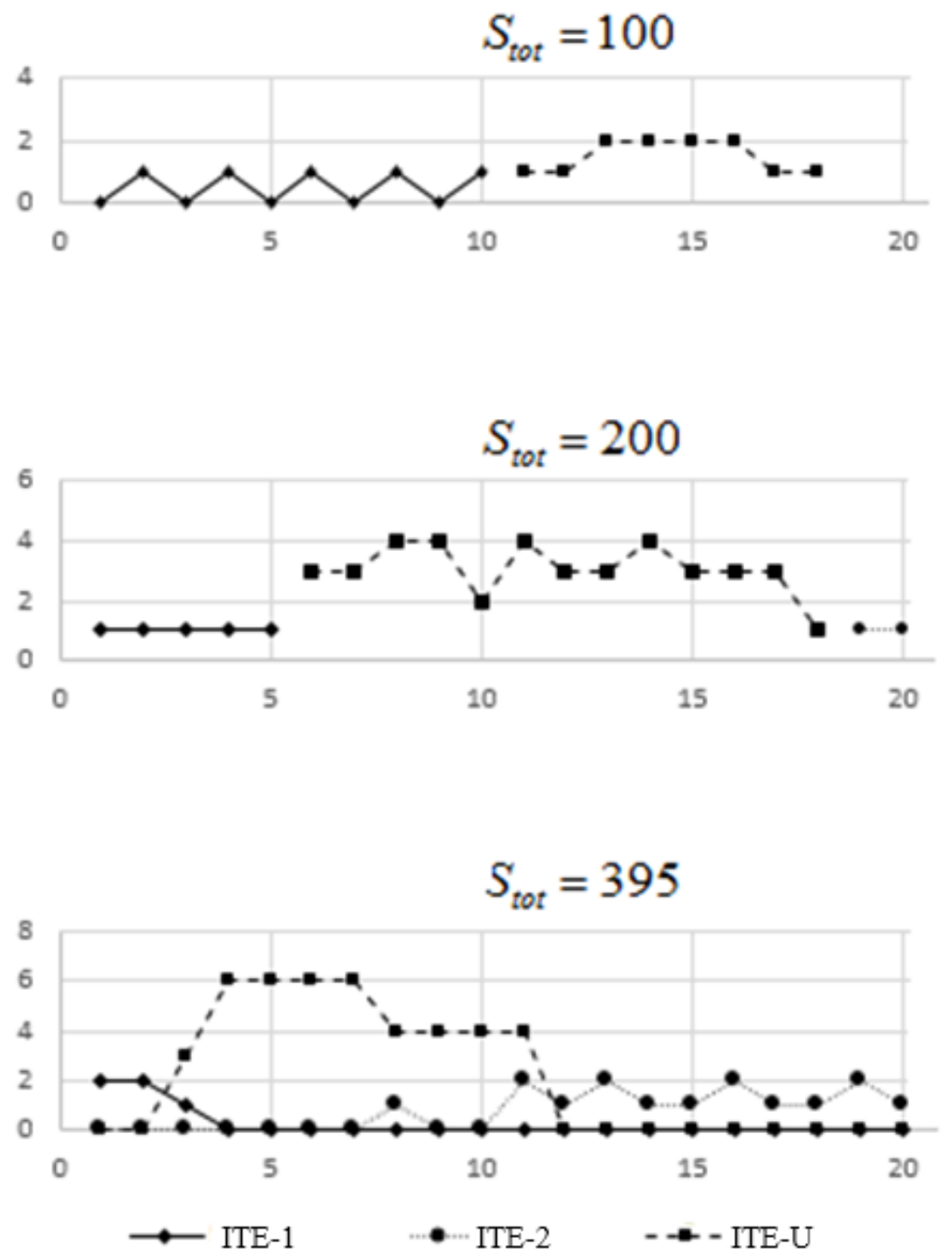

Figure 2. Procurements and modernization of different types of ITE depends on planning period 


\section{Conclusions}

1. Software mathematic support described in the article allows posing and solving linear programing tasks both for general purposes and for integer linear programing. Solution of the latter, especially the tasks of dimensionality, usually requires considerable calculating resources and use of specialized software [8].

2. The experience in making offers for targeted industrial programs and operating control of their implementation has proven the expediency of the model offered in the article. The model based on the solution of integer LPP fits for operating control of purchase and upgrade, as well as for correction of annual plans, whereas long-term (strategic) planning requires standard LPP solutions, with amounts of purchase and upgrade of various ITE types taken as 'managements'.

3. The model developed is a system of linear limitations expressed as equalities (inequalities) and optimum criteria, affords, when required, inclusion of additional qualifying information by changing the vertical dimension of LPP to introduce additional limitations expressed as equalities (inequalities), or be changing LPP horizontal dimension to account for additional ITE types (subtypes).

\section{References}

1. N. Yu. Yas'kova, D. M. Karasik. Programme-oriented methods of construction development, modern format city target programs // the Bulletin]. No. 1. P. 182-186 (2014)

2. V. M. Burenok, R. N. Pogrebnyak, A. P. Skotnikov. Methodology of substantiation of prospects of development of means of armed struggle, General purpose]. Mechanical engineering (2010)

3. A. I. Buravlev, A. A. Nesterov. Methodology for military - economic analysis of the feasibility of procurement of weapons and military equipment // Weapons and the economy. No. 2 (35). P. 83-89 (2016)

4. R. Z. Khayrullin. Targeted management performance indicators Park metrological equipment // Scientific review. No. 10. P. 300-303 (2016)

5. L. S. Pontryagin, V. G. Boltyansky, R. V. Gamkrelidze, E. F. Mishchenko. The mathematical theory of optimal processes]. Moscow. Science (1961)

6. Bryson A., Ho, Y. Applied Optimal Control. Blaisdell Publishing, Walthman, MA (1969)

7. R. P. Fedorenko. Approximate solution of optimal control problems // Moscow. Science (1978)

8. R. Z. Khayrullin. To the optimization of high dimensionality systems using ILOG software components // Vestnik MGSU. No. 8. P. 157-163 (2013) 